Introduction The ISEAN-Hivos Program (IHP) is a regional Global Fund AIDS grant focused on community systems strengthening (CSS) among males having sex with males (MSM) and transgender (TG) organisations in Indonesia, Malaysia, Philippines and Timor Leste. The program in the Philippines since 2011 has provided OD-focused capacity building trainings to MSM/TG CBOs. Ths includes program/ financial management, monitoring and evaluation, among others.

Methods The capacity building series has contributed to strengthening the CBOs organisational systems/procedures. This is evident for more than 25 CBOs whose proposals on innovative HIV, SOGIE and rights-based interventions were granted by IHP through small grants amounting to Php 250 000. Interventions varied from conducting HIV/SOGIErelated awareness campaigns; using theatre as medium for LGBT awareness; establishing a male wellness centre, mobile testing van and BCC-awareness mobile, that contributed to community-led peer education and HIV counselling and testing (HCT) services.

Results Two of the most noteworthy beneficiaries are LoveYouself and Cebuplus. LoveYourself's proposal for HIV awareness IEC video ('Fly Love Yourself') and BCC-awareness mobile ('LoveCar') has contributed in promoting testing services in their community clinics. For Cebuplus, their mobile testing vans and male wellness centre has increased the number of MSM/TG clients reached by their HIV prevention services including HCT. Both have contributed to more than $50 \%$ of the total number of people undergoing HCT in Manila and Cebu, respectively. This has also translated into sustainable partnerships with their local government units and private foundations.

Conclusion This OD-approach model on capacity building for CBOs should continuously be monitored, evaluated, and foster linkage with local government partners and/or private institutions. This CSS intervention has a long-term perspective because it is geared towards the sustainable development of CBOs to continuously advocate and implement community-led HIV, SOGIE and rights-based intervention.

\subsection{SYPHILIS TESTING PRACTICES IN THE AMERICAS: RESULTS OF A REGIONAL SURVEY OF LABORATORIES}

${ }^{1}$ Thuy Trinh, ${ }^{2}$ Freddy Perez, ${ }^{3}$ Minh Luu, ${ }^{1}$ DCal Ham, ${ }^{1}$ Mary Kamb. ${ }^{1}$ Centres For Disease Control And Prevention (CDC), USA; ${ }^{2}$ Pan American Health Organisation (Paho); ${ }^{3}$ Emory University, USA

\subsection{6/sextrans-2017-053264.17}

Introduction Quality of syphilis testing is a critical component of effective STI control programs, including elimination of mother-to-child transmission of HIV and syphilis. To understand syphilis testing policies and practices in the Region of the Americas, the Pan American Health Organisation (PAHO) surveyed directors of national/regional reference and lowerlevel laboratories in its 35 member states.

Methods In 2014, PAHO identified directors of national/ regional reference laboratories and a convenience sample of lower-level laboratories providing clinical diagnostic testing. An electronically-administered survey was used to collect data on tests and algorithms used, quality assurance/quality control (QA/QC) systems, and challenges faced.

Results The 69 participating laboratories, representing 30 $(86 \%)$ PAHO member states, were from Central America $(n=22,32 \%)$, the Caribbean $(n=15,22 \%)$, the Andean $(n=16,23 \%)$ and Southern Cone nations $(n=14,20 \%)$, and North America $(n=2,3 \%)$. Of the 69 laboratories, $41(59 \%)$ were national or regional reference facilities and 28 (41\%) were lower-level facilities ( 25 public and 3 private). Overall 49 (71\%) laboratories (80\% reference, 57\% other) reported using a nationally recommended algorithm for syphilis testing, primarily a non-treponemal screening test and treponemal confirmatory test $(n=35,71 \%)$. Ten laboratories ( 7 reference, 3 other) used an algorithm that did not employ confirmatory testing. Twenty-eight (41\%) laboratories reported using rapid tests (49\% reference, $29 \%$ other). Thirty-three (48\%) could provide results to clinics within 24 hours. Some type of QA/ QC program was reported by 60 (87\%) laboratories $(88 \%$ reference, $86 \%$ other). Of the 69 laboratories, $83 \%$ used daily controls, 64\% routinely maintained equipment, $70 \%$ participated in an external QA program, and 65\% used standard operating procedures. Frequently reported challenges were limited opportunities for staff training (73\%), insufficient equipment $(24 \%)$ and stock outs of reagents and other supplies (55\%).

Conclusion Many reference and clinical laboratories in the region still face challenges in ensuring quality syphilis testing. Many lack adequately trained staff, and some lack sufficient supplies for routine syphilis testing. Several have not yet adopted basic QA/QC practices needed to ensure quality testing. Less than half of the laboratories have adopted rapid testing strategies.

\section{Oral Presentation Session 4}

\section{Women's Health and Prevention}

\subsection{SPECIFIC VAGINAL BACTERIA ARE ASSOCIATED WITH INCIDENT TRICHOMONAS VAGINALIS INFECTION IN WOMEN}

${ }^{1}$ Olamide Jarrett, ${ }^{2}$ Sujatha Srinivasan, ${ }^{2}$ Barbra Richardson, ${ }^{2}$ Tina Fiedler, ${ }^{2}$ Jacqui Wallis, ${ }^{3}$ John Kinuthia, ${ }^{4}$ Walter Jaoko, ${ }^{5}$ Kishor Mandaliya, ${ }^{2}$ David Fredricks, ${ }^{5} \mathrm{R}$ Scott Mcclelland. ${ }^{1}$ University of Illinois at chicago school of medicine, Chicago, USA; ${ }^{2}$ Fred Hutchinson Cancer Research Centre, Seattle, USA; ${ }^{3}$ Kenyatta National Hospital, Nairobi, Kenya; ${ }^{4}$ University of Nairobi, Nairobi, Kenya; ${ }^{5}$ University of Washington, Seattle, USA

\subsection{6/sextrans-2017-053264.18}

Introduction We tested the hypothesis that vaginal microbiota influence women's susceptibility to $T$. vaginalis (TV) acquisition.

Methods We conducted a nested case-control study of 25 episodes of TV infection using vaginal samples collected 30-60 days prior to infection in 18 HIV-negative women and 50 incidence-density matched controls. Broad-range 16S rRNA gene PCR was used to measure total vaginal bacterial load. Deep sequencing was applied to 18 first episodes of TV infection and 36 matched controls to measure bacterial diversity (Shannon index) and species richness (Chao-1 index). Bacterium-specific quantitative PCR was performed for Lactobacillus crispatus, Prevotella amnii, Sneathia sanguinegens, Dialister species Type 2, Bacterial Vaginosis Associated Bacterium 1 (BVAB-1), and Mageeibacillus indolicus for all TV infection cases and controls. The associations between Shannon and Chao- 1 indices and TV acquisition were evaluated using logistic regression. Generalised estimating equations with logit link 